\title{
Integrable Systems of Partial Differential Equations Determined by Structure Equations and Lax Pair
}

\author{
Paul Bracken \\ Department of Mathematics, \\ University of Texas, \\ Edinburg, TX
}

78541-2999

\begin{abstract}
It is shown how a system of evolution equations can be developed both from the structure equations of a submanifold embedded in three-space as well as from a matrix $S O(6)$ Lax pair. The two systems obtained this way correspond exactly when a constraint equation is selected and imposed on the system of equations. This allows for the possibility of selecting the coefficients in the second fundamental form in a general way.
\end{abstract}

MSC: 53C44, 58A10, 58H5 


\section{Introduction.}

There are some remarkable relationships between certain classes of partial differential equations and the geometry of surfaces, or submanifolds, immersed in three-dimensional space which correspond to them [1]. Moreover, a great many partial differential equations which are of interest to study and investigate due to the role they play in various areas of mathematics and physics are included in this category [2-4]. It must also be stated that these equations typically result as the integrability condition of a linear system or pair of linear equations usually referred to as a Lax pair. The cases of constant total or Gaussian curvature as well as mean curvature have been studied extensively. It might then be asked what can be said with regard to the more general cases in which one or both of the curvatures of the submanifold is or are not constant.

Here we would like to ask what can be said about the correspondence between partial differential equations which can be obtained from a linear pair of matrix equations as well as from the structure equations for a two-dimensional submanifold or surface which is embedded in threedimensional space such that the coefficients of the second fundamental form are left arbitrary at first. This will turn out to produce a general relationship between a system of partial differential equations on the one hand and an associated surface on the other. To state this another way, it is proposed to see how previous results [5-6] can be generalized to situations in which the curvatures of the submanifold do not turn out to be identically constant. It will be found here that the moving frame approach will permit the calculation of the basic fundamental forms which are sufficient to determine the submanifold once the one-forms in the structure equations are defined. It will be seen that the system of equations which are produced by the structure equations under a particular specification of the basic one-forms can be exactly duplicated by defining the form of a particular linear matrix Lax pair, up to specifying a single constraint on some of the functional quantities which appear. These equations will be given for a specific choice of one-forms, although other choices may be possible. This work serves to generalize the $S O(3)$ Lax pair which was produced in [5]. Generalizations of the $S O(2,1)$ Lax pairs have also been done and will be reported later.

\section{Structure Equations and Differential Forms.}


Suppose $\mathbf{x}: M \rightarrow \mathbb{R}^{3}$ is a smooth surface in $\mathbb{R}^{3}$. Choosing local coordinates $t$ and $x$ in a coordinate neighborhood $U$ in $M$, the surface can be expressed by the parametrized equations $x^{i}=x^{i}(x, t), 1 \leq i \leq 3$. Choose a Darboux frame $\left(x, e_{1}, e_{2}, e_{3}\right)$ on $M$ such that $e_{1}$ and $e_{2}$ are tangent to $M, e_{3}$ is normal to $M$ and the orientation of $\left(e_{1}, e_{2}, e_{3}\right)$ is the same as a chosen orientation of $\mathbb{R}^{3}$. Suppose the corresponding relative components for the frame field are written $\omega_{i}, \omega_{i j}$, then $[7-8]$

$$
\begin{aligned}
& d \mathbf{x}=\omega_{1} e_{1}+\omega_{2} e_{2}, \quad \omega_{3}=0, \\
& d e_{j}=\omega_{j i} e_{i}, \quad \omega_{i j}+\omega_{j i}=0,
\end{aligned}
$$

where $\omega_{i}, \omega_{i j}$ are differential 1 -forms of the parameters $t, x$. The structure equations are

$$
\begin{gathered}
d \omega_{1}=\omega_{2} \wedge \omega_{21}, \quad d \omega_{2}=\omega_{1} \wedge \omega_{12}, \\
\omega_{1} \wedge \omega_{13}+\omega_{2} \wedge \omega_{23}=0, \\
d \omega_{12}=\omega_{13} \wedge \omega_{32}, \quad d \omega_{13}=\omega_{12} \wedge \omega_{23}, \quad d \omega_{23}=\omega_{21} \wedge \omega_{13} .
\end{gathered}
$$

By Cartan's Lemma, based on (2.4), it follows that there exist functions $h_{i j}$ such that

$$
\omega_{13}=h_{11} \omega_{1}+h_{12} \omega_{2}, \quad \omega_{23}=h_{21} \omega_{1}+h_{22} \omega_{2}, \quad h_{12}=h_{21} \equiv h .
$$

Now equations (2.5) are the Gauss equation and the Codazzi equation of $M$. The first and second fundamental forms can be obtained from the forms which appear in this system. These essentially determine the surface up to rigid motions. The first, second and third fundamental forms for $M$ are given by,

$I=d \mathbf{x} \cdot d \mathbf{x}=\left(\omega_{1}\right)^{2}+\left(\omega_{2}\right)^{2}, \quad I I=-d \mathbf{x} \cdot d e_{3}=\omega_{1} \omega_{13}+\omega_{2} \omega_{23}, \quad I I I=d e_{3} \cdot d e_{3}=\left(\omega_{13}\right)^{2}+\left(\omega_{23}\right)^{2}$.

The mean curvature and total curvature of $M$ are determined by $h_{i j}$ and are both independent of the choice of Darboux frame with

$$
H=\frac{1}{2}\left(h_{11}+h_{22}\right), \quad K=h_{11} h_{22}-h_{12}^{2} .
$$


Now let us specify the forms, substitute them into the structure equations and simplify to see what results without at first specifying the quantities $h_{i j}$. When the coefficients of the forms are subsequently given in terms of one or more unknown functions $\varphi_{j}(x, t)$ as well, a system of partial differential equations in terms of the $\varphi_{j}$ will be seen to emerge,

$$
G_{i}\left(\varphi_{j}, \varphi_{j, x}, \varphi_{j, t}, \cdots\right)=0
$$

The key idea is that these partial differential equations will arise both from the structure equations as well as from a matrix Lax pair. This means an important aspect of integrability is met automatically. Of course, we keep to a pair of variables $x, t$ since the Lax pair will depend on two variables. Keeping the level of complexity to a low level, a system of one-forms are defined $\omega_{i}$ which depend on six functions $u_{i j}$ and $v_{i j}$ such that the forms are given by

$$
\omega_{1}=u_{12} d t+v_{12} d x, \quad \omega_{2}=u_{13} d t+v_{13} d x, \quad \omega_{3}=0
$$

It is the coefficients of the forms in (2.10), $u_{i j}$ and $v_{i j}$, that depend on $\varphi_{i}$ which specify the differential equations. The forms which specify the connection are

$$
\omega_{12}=u_{23} d t+v_{23} d x
$$

and $\omega_{13}, \omega_{23}$ which can be written down using Cartan's Lemma given $\omega_{1}$ and $\omega_{2}$ from (2.10) are

$$
\omega_{13}=\left(h_{11} u_{12}+h u_{13}\right) d t+\left(h_{11} v_{12}+h v_{13}\right) d x, \quad \omega_{23}=\left(h u_{12}+h_{22} u_{13}\right) d t+\left(h v_{12}+h_{22} v_{13}\right) d x
$$

Differentiating the forms and substituting into (2.3) and (2.5), the results can be summarized as follows

$$
\begin{gathered}
u_{12, x}-v_{12, t}+u_{23} v_{13}-u_{13} v_{23}=0, \\
u_{13, x}-v_{13, t}+u_{12} v_{23}-u_{23} v_{12}=0, \\
u_{23, x}-v_{23, t}+\left(h_{11} h_{22}-h^{2}\right)\left(u_{13} v_{12}-u_{12} v_{13}\right)=0, \\
\left(h_{11} u_{12}+h u_{13}\right)_{x}-\left(h_{11} v_{12}+h v_{13}\right)_{t}+u_{23}\left(h v_{12}+h_{22} v_{13}\right)-v_{23}\left(h u_{12}+h_{22} u_{13}\right)=0 \\
\left(h u_{12}+h_{22} u_{13}\right)_{x}-\left(h v_{12}+h_{22} v_{13}\right)_{t}+v_{23}\left(h_{11} u_{12}+h u_{13}\right)-u_{23}\left(h_{11} v_{12}+h v_{13}\right)=0 .
\end{gathered}
$$


This is the system of equations which results from the structure equations.

\section{Equations Determined by a Linear System.}

It will now be seen how system of equations (2.13) can be obtained from an $S O(6)$ matrix Lax pair. It is to be required that the two linear systems

$$
\Phi_{t}=U \Phi, \quad \Phi_{x}=V \Phi
$$

generate system (2.13) when the zero curvature condition is enforced. The integrability condition which follows from this set of linear equations (3.1) in terms of $U$ and $V$ takes the form

$$
U_{x}-V_{t}+[U, V]=0
$$

It will be shown that there is at least one way to get (2.13) by picking $U, V$ appropriately. Suppose we take $U=U_{1} \oplus U_{2}$ and $V=V_{1} \oplus V_{2}$ of the form

$$
U=\left(\begin{array}{cc}
U_{1} & 0 \\
0 & U_{2}
\end{array}\right), \quad V=\left(\begin{array}{cc}
V_{1} & 0 \\
0 & V_{2}
\end{array}\right),
$$

so the compatibility condition (3.2) reduces to $U_{i, x}-V_{i, t}+U_{i} V_{i}-V_{i} U_{i}=0$ for $i=1$, 2 . To specify the submatrices $U_{1}$ and $V_{1}$, we take the following $S O(3)$ matrices,

$$
U_{1}=\left(\begin{array}{ccc}
0 & u_{12} & u_{13} \\
-u_{12} & 0 & u_{23} \\
-u_{13} & -u_{23} & 0
\end{array}\right), \quad V_{1}=\left(\begin{array}{ccc}
0 & v_{12} & v_{13} \\
-v_{12} & 0 & v_{23} \\
-v_{13} & -v_{23} & 0
\end{array}\right) .
$$

The submatrices $U_{2}$ and $V_{2}$ are given by

$$
\begin{gathered}
U_{2}=\left(\begin{array}{ccc}
0 & h_{11} u_{12}+h u_{13} & h u_{12}+h_{22} u_{13} \\
-h_{11} u_{12}-h u_{13} & 0 & u_{23} \\
-h u_{12}-h_{22} u_{13} & -u_{23} & 0
\end{array}\right), \\
V_{2}=\left(\begin{array}{ccc}
0 & h_{11} v_{12}+h v_{13} & h v_{12}+h_{22} v_{13} \\
-h_{11} v_{12}-h v_{13} & 0 & v_{23} \\
-h v_{12}-h_{22} v_{13} & -v_{23} & 0
\end{array}\right) .
\end{gathered}
$$

Substituting (3.4), (3.5) into (3.3) and then using (3.3) in (3.2), it is a straightforward calculation to show that (3.2) is satisfied provided the following system holds,

$$
u_{12, x}-v_{12, t}+u_{23} v_{13}-u_{13} v_{23}=0
$$




$$
\begin{gathered}
u_{13, x}-v_{13, t}+u_{12} v_{23}-u_{23} v_{12}=0, \\
u_{23, x}-v_{23, t}+u_{13} v_{12}-u_{12} v_{13}=0, \\
u_{23, x}-v_{23, t}+\left(h_{11} h_{22}-h^{2}\right)\left(u_{12} v_{12}-u_{12} v_{13}\right)=0, \\
\left(h_{11} u_{12}+h u_{13}\right)_{x}-\left(h_{11} v_{12}+h v_{13}\right)_{t}+u_{23}\left(h v_{12}+h_{22} v_{13}\right)-v_{23}\left(h u_{12}+h_{22} u_{13}\right)=0, \\
\left(h u_{12}+h_{22} u_{13}\right)_{x}-\left(h v_{12}+h_{22} v_{13}\right)_{t}+v_{23}\left(h_{11} u_{12}+h u_{13}\right)-u_{23}\left(h_{11} v_{12}+h v_{13}\right)=0,
\end{gathered}
$$

The matrices $U$ and $V$ therefore reproduce all five equations in (2.13) which result from the structure equations except there is a additional equation. The third and fourth equations in (3.6) appear in two different forms. In fact, these two different forms can be exactly matched by introducing a constraint, which can be chosen in two ways. These two equations will match provided that

$$
\left(u_{12} v_{13}-u_{13} v_{12}\right)\left(h^{2}-h_{11} h_{22}+1\right)=0
$$

Clearly, this can be satisfied in two different ways. First of all, it can be satisfied by putting a constraint on four of the functions $u_{i j}$ and $v_{i j}$, namely, $u_{12} v_{13}-u_{13} v_{12}=0$. Using this, no restrictions need be placed on the coefficients of the second fundamental form. Another way to satisfy (3.8) is to put a constraint on the set of functions $h_{i j}$, namely, $h_{11} h_{22}-h^{2}=1$. This result states that the total curvature must be one, beyond that, the quantities appearing in it can be arbitrary.

If the constraint on $u_{i j}, v_{i j}$ is substituted in the third and sixth equations in (3.6), these two equations reduce to the common expression $u_{23, x}-v_{23, t}=0$. Moreover, $v_{13}=u_{13} v_{12} / u_{12}$ must be substituted into the remaining equations in (3.6), and no external restriction need be placed on the second fundamental form. On the other hand, if we suppose the coefficient matrix of the second fundamental form has elements which satisfy $h_{11} h_{22}-h^{2}=1$, the third and sixth equations of (3.6) reduce to a form with a common structure, so these five equations exactly match (2.13). These five equations can be solved as a system.

Notice that these results imply that the pair of three by three matrices which form the matrices (3.3) are not completely independent of each other or completely decoupled at the end.They are in 
a way coupled together by means of the constraint which is imposed. Suppose $h_{11}=h_{22}=1$, then the final pair of equations in (2.13) go into two of the first three equations. As a short example for this choice of constraint, if we let $u_{13}=v_{12}=0, u_{12}=\cos (\varphi / 2)$ and $v_{13}=\sin (\varphi / 2)$ then (2.13)-(3.6) become

$$
\left(\cos \left(\frac{\varphi}{2}\right)\right)_{x}+u_{23} \sin \left(\frac{\varphi}{2}\right)=0, \quad-\left(\sin \left(\frac{\varphi}{2}\right)\right)_{t}+\cos \left(\frac{\varphi}{2}\right) v_{23}=0, \quad u_{23, x}-v_{23, t}-\cos \left(\frac{\varphi}{2}\right) \sin \left(\frac{\varphi}{2}\right)=0
$$

Solving for $u_{23}$ and $v_{23}$ from the first pair and substituting into the third, we obtain that $\varphi$ satisfies the integrable equation $\varphi_{t t}-\varphi_{x x}=-\sin (\varphi)$.

\section{References.}

[1] C. Rogers and W. F. Schief, Bäcklund and Darboux Transformations, Geometry and Modern Applications in Soliton Theory, Cambridge Texts in Applied Mathematics, Cambridge University Press, (2002).

[2] G. Chaohao, Soliton Theory and Its Applications, Springer-Verlag, Berlin (1995).

[3] A. S. Fokas and I. M. Gelfand, Surfaces on Lie Groups, on Lie Algebras, and Their Integrability, Commun. Math. Phys., 177, (1996), 203-220.

[4] A. S. Fokas, I. M. Gelfand, F. Finkel and Q. Liu, A formula for constructing infinitely many surfaces on Lie algebras and integrable equations, Selecta Mathematica, New Series, 6, (2000), $347-375$.

[5] P. Bracken, Partial Differential Equations Which Admit Integrable Systems, Int. J. of Pure and Applied Math., 43, (2008), 408-421.

[6] P. Bracken, Integrable Systems Determined by Differential Forms for Moving Frames on Immersed Submanifolds, Int. J. Geometric Methods in Mod. Physics, 5, (2000), 1041-1049.

[7] S. S. Chern, W. H. Chen and K. S. Lam, Lectures on Differential Geometry, World Scientific, Singapore, (1999).

[8] H. Cartan, Differential Forms, Dover Pub., Mineola, NY, (2006). 